\title{
ONLINE PURCHASING PLATFORM USING CROWD SOURCING WITH IMPROVISATION OF CLASSIFICATION ACCURACY
}

\author{
P. Gokulakrishnan ${ }^{1}$ D. Suresh ${ }^{2}$ S. Satheesbabu ${ }^{3}$ \\ ${ }^{1}$ Professor, PSNA College of Engineering and Technology, Dindigul, India \\ ${ }^{2}$ Professor, PSNA College of Engineering and Technology, Dindigul, India \\ ${ }^{3}$ Associate Professor, PSNA College of Engineering and Technology, Dindigul, India \\ gokulakrishnan@psnacet.edu.in
}

\begin{abstract}
:
Crowd-sourcing is a prototype where persons cum organisations acquire facts such as ideas, micro-tasks, financial, vote casting associated to items and offerings from individuals of large, open and rapidly-evolving nature. It entails utilization of web acquired and distribute work between members to get a collective result. The software of classification tasks in crowd-sourcing is a counter step due to the inclined reputation of crowd-sourcing market. Dynamic Label Acquisition and Answer Aggregation (DLTA) crowd-sourcing framework accomplishes the classification assignment in a promising manner. But most of the current works are now not in a position to supply an budget allocation for labels due to the fact they do not make the most the Label inference and acquisition phase. In addition, label mismatch and multi-label tasks are the different issues encountered in the current works. To overcome, it is proposed to undertake Random Forest Algorithm (RFA) for classification in crowd-sourcing. The goal of this work is to enhance the crowd-sourcing classification task efficiency with Dynamic Resource Algorithm. RFA is activated by means of developing a multitude of decision tree at training time and consequences with the training and it applies a bagging approach to produce the last end result with more accuracy.
\end{abstract}

Index terms: Crowd-sourcing classification, Random Forest Algorithm, Bagging Techniques, Label Acquisition, Multi-label tasks.

\section{I.INTRODUCTION}

Crowd-sourcing is an important platform to tackle tasks utilized by using heaps of most common employees or customers (i.e., the crowd). Crowd-sourcing is enticing a team or crowd to attain a company goal, which may also be efficiency, innovation, or hassle solving. Public crowd-sourcing platforms, like Amazon Mechanical Turk (MTurk), Crowd Flower and Up-work are exercising the crowd-sourcing market to ail persons cum companies for outsourcing their jobs and methods to a disbursed body of workers for digital assignment performance.Crowdsourcing additionally profitsinformation ad ministration purposes like data cleansing, statistics integration, statistics construction. 
Consider the entity resolution as associate degree example, believe a consumer (called the "requester") elements a set of objects and wants to search out the objects that discuss to an equal entity, possibly victimization totally exceptional names. Though this drawback has been studied for many years, historic algorithms rectangular measure nonetheless off from excellent.

To this finish, the requester 1 st patterns the tasks (e.g., a challenge for every mix of objects that asks team of workers to factor whether or not the two objects speak to an equal entity.) or no longer Then the requester publishes the tasks on a crowd sourcing platform like AMT Crowd workforce who rectangular measure inclined to function such tasks(typically for pay or any other reward) settle for the tasks, reply them and post the reply and file them to the requester. The platform collects the solutions and provides to the requester. Because the crowd has discourse information and function ability, crowd sourced entity decision will enhance the standard. There are several other problems in crowd-sourced data management as depicted in Figure 1.

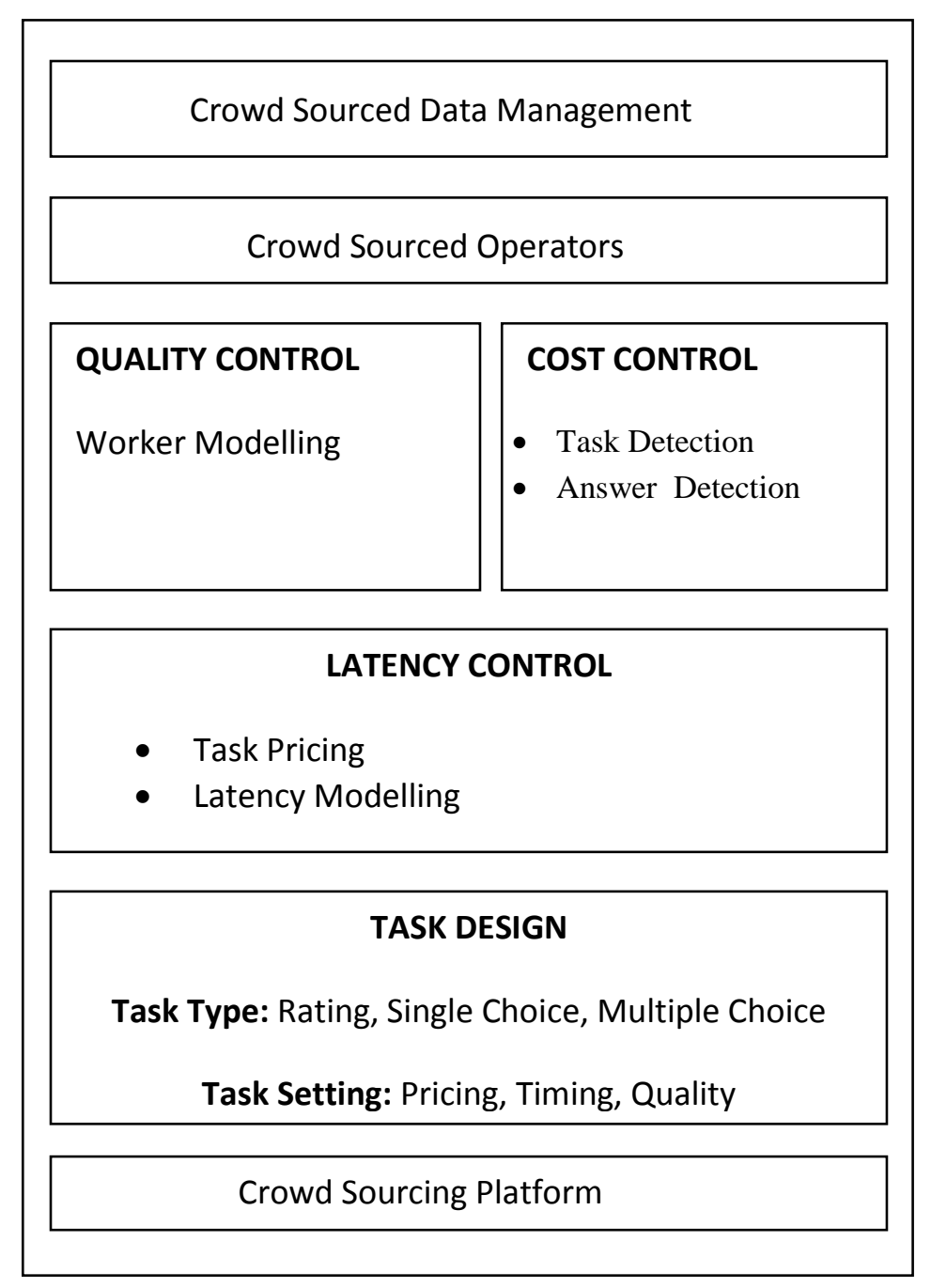

Figure 1: Overview of Crowd-sourced Data Management Internal Control: 
Crowd-sourcing should yield comparatively low-quality effects or possibly noisy. For instances, a malicious worker may want to on cause supply incorrect answers. Staff should have definitely extraordinary stage of experience, related a primitive worker is additionally incapable of conducting sure tasks.

To acquire top quality, tolerate the crowd mistakes and infer excessive high-quality consequences from strident answers. The important step of interior manage is to signify an employee's pleasant (called employee modelling). Then help the general mannequin of staff, there are many strategies for enhance quality. Eliminate the low pleasant group of workers (called worker elimination), assign a assignment to a couple of team of workers and mix their solutions (called reply aggregation), or assign a assignment to relevant workforce (called venture assignment).

\section{Price Management:}

The team isn't free, and if there are massive numbers of tasks, crowd-sourcing may additionally be pricey. For instance, in entity resolution, if there are ten thousand objects, there will be concerning fifty million pairs. Though the well worth per attempt in one cent, it nonetheless takes hundreds of thousands of cash. There are many high quality fee manage techniques.

The fundamental is pruning, that makes use of computer algorithm to get rid of some surplus tasks then make use of the crew to reply totally the obligatory tasks. The 2 nd is venture desire that prioritizes these tasks to crowd source. The 0.33 is reply deduction that crowd sources the set of tasks and supported the solutions accumulated from the group; deduce the consequences of choice tasks. The fourth is sampling, that samples a set of tasks to crowd source. There additionally some specialised fee manipulate methods specifically designed to optimize for precise operators.

\section{Latency Management:}

Crowd answers should incur immoderate latency for many reasons: for instance, man strength is additionally distracted or untouchable, the tasks would possibly now not be attractive to ample staff, or the tasks it is now not might also be challenging for many staffs. If the requester contains a time constraint essential to alter the latency. There are many strategies for latency management, main is valuation. Typically a higher cost attracts a lot of group of workers and may scale again the latency. The 2nd is latency modelling.

There are specially two latency models: the spherical mannequin and utilized mathematical model. (a) The spherical mannequin leverages the notion that tasks possibly printed in more than one rounds. If there are adequate team of workers in crowd-sourcing platform, the latency of respondent project in each and every spherical may additionally be concept to be steady time. Therefore the latency is sculptured due to the fact the range of rounds. (b) The utilized mathematical mannequin is moreover wont to be mannequin latency that leverages the accumulated facts from preceding crowd-sourcing tasks to create utilized arithmetic mannequin that may also seize the worker's factor in time, the completion time, etc. These 
delivered fashions will then be wont to predict and can also be regulated for anticipated latency. Three greater components of crowd sourced know-how administration are: mission style, crowd sourced operator style, and optimization.

Given a mission (e.g. entity resolution), venture fashion goals to fashion fantastic project varieties (e.g. making a YES/NO questions related asking workforce to select out an answer). Task style moreover need to set the residences of tasks, that is deciding the cost, placing the time constraint, and deciding on quality-control ways.

\section{II.RELATED WORKS}

Libon Zheng proposed a DLTA Framework for Dynamic Crowd sourcing Classification Tasks [1]. The framework proceeds in a sequence of spherical robin (GRR) and also with label inference and label acquisition. For every spherical it accumulated the reply of preceding rounds and analyzes to operate ideal price range allocation and promises the resultant question to the crowd. The primary gain of the gadget is to advise a generative mannequin for label collections and additionally depict the corresponding techniques for label inference and finances allocation and experimental consequences exhibit that in contrast with present methods. DLTA Approach does now not take advantage of the label inference and acquisition phase. This outcomes in incapability of making a suited price range allocation for labels. Label mismatch, Multi-label assignment are some of the extra troubles confronted in the DLTA Framework.

Mohammad Asghari and Cyrus Shahabi proposed an On-line Task Assignment in Spatial Crowd-sourcing[2]. Auction based totally algorithm (ABA) is one in which break up the scheduling tasks and matching between the spatial crowd server and workers. Spatial crowdsourcing entails hundreds of thousands of people and tasks and additionally allocate the assignment to a employee based totally on matching project to employee and computing a time table for every worker. If every mission is carried out instantly, the mission is scheduling the task. The On-line Task Assignment designed for the workers, who journey to a single location. But the different utility needs a employee journey to greater than one place. For instance in Uber software, the employee has to choose a passenger in one vicinity and drop them in different place. So, it wishes a prolonged auction-based framework.

Megan K.O' Brien and Christian Poellabauer proposed a Detecting Errors in Crowd-sourcing Smart Phone Sensor Data[3], Which furnish the information line for creating advantageous methods to become aware of and dispose of the label blunders in clever smart-phone sensor data. It works on label mistakes the usage of Supervised Learning Algorithm known as Data Corruption and Ensemble algorithm (DCE) to restriction the era of false outputs in the presence of label errors. The label error is manifest if the label is corrupted accidently or deliberately. It solves the difficulty by using Supervised Learning for public sale recognition. It converts education scheme into 4 corporations of sub classifiers. The sub classifiers does no longer specify the following attributes

a) The simulated information set may additionally no longer entirely mirror actual world label errors. 
b) The mannequin of the router ought to be flawed.

c) The statistics set has an imbalanced distribution.

d) Small records set can also restrict the classifier's performance.

Ting Wu et al proposed a Object Identification with Pay-As-You-Go crowd-sourcing [4]. It deploys the new crowd-sourcing paradigm for OI tasks, named Adaptive Worker Assignment. It tackle the two problems

a) Assigning the questions to the fine people with the consideration of the Pay-AsYou-Go charge scheme.

b) Design termination standards and affirm the crowd sourced outcomes from preceding workers.

The first trouble solved by means of near-optimal algorithm which determines a near-optimal set of people to be crowd sourced, and grasping method used for pick and crowd sources a near-optimal set of workers. For the $2 \mathrm{~d}$ trouble we want to determine, when to cease asking questions related to every worker. To locate this majority balloting for aggregating crowdsourced records and imposed a threshold so that terminate crowd-sourcing. Each worker terminates the process, solely if the chance of reply is being right and additionally no much less than the given threshold.

Xiano Duan and Keisha Tajima proposed a Hierarchical Reorganization For Improving The Classification Accuracy in crowd-sourcing[5]. It focuses on the accuracy of multi-class classification tasks in crowd sourcing through Worker allocation algorithm (WAA). It understand every mission into hierarchical classification tasks and assign the employees to fabulous sub-tasks in the hierarchy. The fundamental goal of the work is to assign employees to tasks they are desirable at. Because, distinct employees are excellent at unique categories. It converts flat classification tasks into hierarchical classification tasks and assign people to sub-tasks that they are excellent at. It is solely the notion for enhancing classification accuracy. So, the validation of the thinking is accomplished via actual mission execution on the crowd-sourcing platform.

Antonella Frisiello et al described a Gamified crowd-sourcing for Disaster Risk Management[6]. It proposes a Gamified Strategy for crowd sourced catastrophe hazard administration offerings aimed to enlarge awareness, engagement, and alternate human beings behaviours. It is aimed to enhance the citizen engagement in the direction of subjects associated to co-operative environmental monitoring, danger cognizance and herbal hazards. The desires of Gamified approach is citizen's awareness, citizen's engagement in reporting , file validation. It consists of 3actors (players, spectators, observers). The evaluation applies the major substances given through present day literature, and stimulating an energetic mindset and self-protection behaviours. Here, the video display units records series for herbal dangers via crowd-sourcing, which minimize influences in phrases of human and financial losses in case of herbal disasters. The future work consists of implementation and contrast of 
gamified cellular utility for crowd sourcing. The comparison is accomplished by way of consumer experience, engagement and first-rate of the contents produced.

Raffaella Guida et al proposed a Remote Sensing and Crowd-sourcing [7]. In this work crowd-sourcing is expressed as faraway sensing assignment to manipulate water fine in Africa, Where the truths are accrued from the education human beings in nearby communities. The Remote Sensing Algorithm (RSA) or Detection algorithm is used to validate the floor truths. It proceeds in two perspectives:

a) Monitor the water quality of its predominant reservoir.

b) Monitor the incidence of water borne ailments in children.

In contrast evaluation no longer all the statistics have been returned. The subsequent step is correlate the consequences on high-quality of water pattern and solutions to the questionnaires submitted by using the children.

Hien et al outline a Acquisition and Analysis of Data For Disaster Response [8]. The growing recognition of cell devices, crowd-sourcing statistics series and evaluation emerged as a scalable solution. The Acquisition and Analysis segment tackle the two challenges.

a) Transmission below band width shortage induced via broken verbal exchange community and prioritizing the visible statistics collection.

b) Analyze the received facts in a timely manner.

The analytical mannequin is supplied to quantify the visible consciousness of a video primarily based on its meta data. For obtaining most applicable records underneath bandwidth constrains, leads to visible focus maximization problem. Future work consists of learn about of crowd-sourcing applied sciences which consists of each the analysts at the command centre and the managed employees at the catastrophe website to reply some open questions, justifies who to ask and the place to acquire the information in disasters.

Shyamala Ramachandran and Sasireka introduced Descriptive Study and Analysis of Crowdsourcing Technique [9]. It entails cell crowd-sourcing methods like Task Assignment Based Methods, Group Based Requirement System, and Green Mobile Crowd Sensing Based Techniques. Descriptive Study and Analysis of Crowd-sourcing Technique grant an elaborative evaluation and dialogue are made with assessment metrics, utilized datasets, employed methods, implementation and power consumption, guide year.

Eventually analyze the lookup gaps and problems of a number cellular crowd-sourcing techniques. The obstacles of Task Assignment Based Technique is best of provider metric is now not considered. The foremost undertaking in Green Mobile Crowd Sensing Based Technique is does no longer measure the strength saving for geographical sensors. The essential assignment in different Mobile Crowd Sensing-Techniques is prior Knowledge and correct mannequin is now not considered. The future work includes, in addition scope for cell crowd sensing strategies via thinking about problems and lookup gaps. 
C.Bielski et al proposed a Coupling Early Warning Services, Crowd-sourcing and Modelling for Improved Decision Support and Wildfire Emergency Management[10]. This describes the Wildlife Monitoring for Emergency Management System in Wildlife Disasters. It is developed for European Forest Fire Disasters, Based on the integration of data from special sources, statistics processing chains and choice help systems. The device graph is applied with the aid of Data Processing and Analysis Chain phase.

The facts administration is managed through Processing Description phase. It consists of climate forecasting, the furnace climate index, monitoring hotspots, affirmation of wildfire, wildfire dangers now casting and forecasting, wildfire chance affect mapping, wildfire catastrophe selection support, burned location mapping, citizen involvement. The future work consists of new algorithms and handy statistics sources, which without difficulty adopted and ingested to enhance Wild Fire Crisis Management.

Ning $\mathrm{Xu}$ et al proposed a Ontological Bagging Approach for Image Classification of Crowd sourced Data[11]. It proceeds via ontological bagging algorithm(OBA), it works with the aid of studying the most susceptible attributes for unique semantic stages by using a couple of occasion studying and the error propagation of hierarchical classifiers are decreased through bagging idea. The major benefit is, it learns discriminative aspects on every degree of ontology the usage of a couple of occasion learning, and classifies classes from coarse to best semantic grains primarily based on these features, Which mimics the human visible system. The future work is, to consider the work on other realistic picture records sets.

Markus herb et al proposed a Crowd Sourced Semantic Edge mapping for self sufficient motors [12]. It proceeds by using a Novel approach to derive a specific excessive definition maps by using crowd-sourcing information the usage of commodity sensors. It makes use of multi-session characteristic based totally visible SLAM to align sub maps recorded through person motors on a central backend server. The future work is to consider a technique on actual world data, which include high stage of element and metric accuracy.

Sumit Mishra et al proposed a Non-dominated sorting for Worker Selection in Crowd Sourced platforms[13]. The purpose of the work is to function a non-dominated sorting of the employees primarily based on the requirement. From this set of ordered employees domination remember is used to choose the exceptional set of people that can operate the task. The future work consists of to generalize the thinking of band with the aid of incorporating the people of the first to ok -1 th degree whilst deciding on the employees from kth level.

Xiangpeng et al proposed a [14], which improves the navigation of cars in city areas and collects the high amount of records with the aid of on-board and infrastructure based totally sensors for consider site visitors community statuses. The foremost goal is to depict a realtime route planning algorithms, which determines the satisfactory trajectory in a real-time relies upon on the accepted information inputs. The future work is to focal point on designing facts processing approach at the back-end stage to deal with the unavailability and mistakes of the said data. 


\section{III.PROPOSED SYSTEM}

Crowd-sourcing methods are focuses on enhancing the efficiency and classification accuracy of crowd-sourcing applications. The Figure two suggests the Architecture of the proposed work. Architectural works represents set of concepts, which consists of their principles, factors and components. Proposed machine has three modules, they are:
A. Pre-Processing module
B. Feature Extraction module
C. Classification module

\section{A. PRE-PROCESSING}

Creating the platform for on-line buying is the initial process. Pre-processing offers with growing

- Process of Admin

- Registration of User

- Purchasing of Product

- Getting reviews.

\section{Process of admin:}

Admin performs a principal position and has the accountable of including dependable merchandise as per corresponding categories. User can purchase their required merchandise and can publish the assessment about the products. Admin has all privileges including the ability to block the user's account and add products.

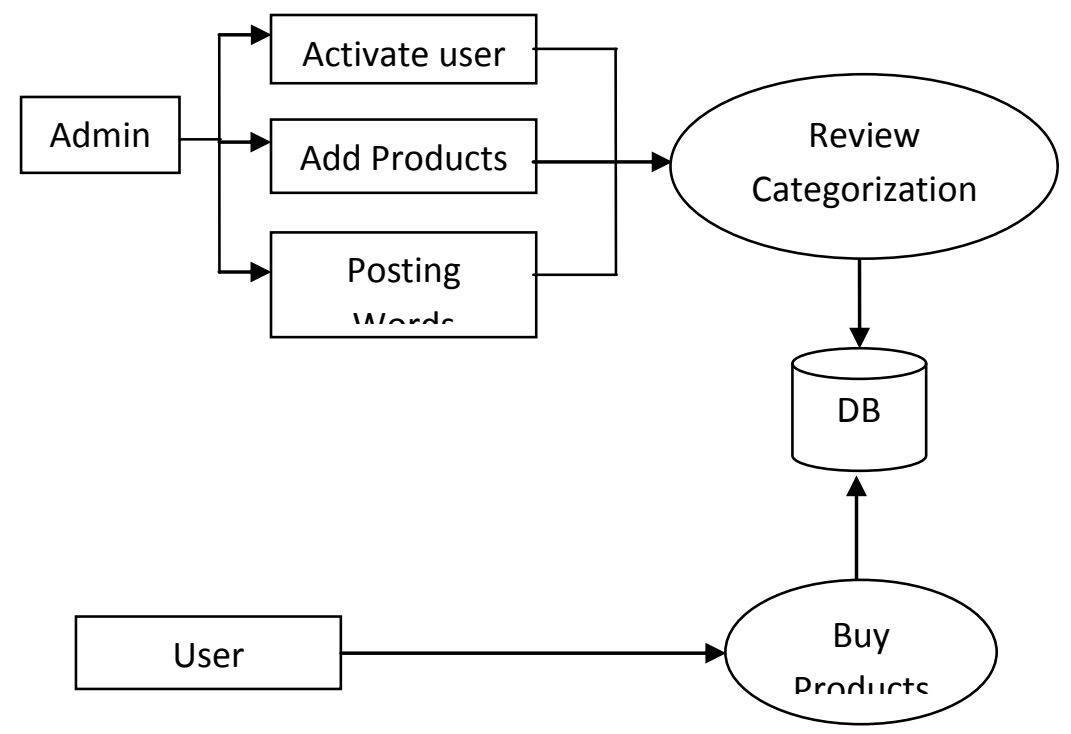

Figure 2:Architecture of Proposed System 


\section{Registration of User:}

User Registration module requires credential of the user like username, password,etc.

\section{Purchasing of Product:}

Product Purchase module allows the user to view the listed items that are provided by the admin.

\section{Getting Reviews:}

User can post a review after bought a product, in terms of realizing the features of the product.

\section{B.FEATURE EXTRACTION}

Feature Extraction can be done by C4.5 (J48) Algorithm, Which defines the product added by the admin, is belonging to Electronics or Appliances or Accessories, etc. Also provide the sales platform includes getting Reviews. C4. 5 works by creates decision tree using the expected values of the class.

\section{C.CLASSIFICATION}

In classification, the getting reviews are classified into positive and negative comments. Classification is achieved by Random Forest Algorithm, Which is operates by constructing a multitude of decision tree at training time and also results the mode of classes.

\section{Working Principle:}

Random Forest applies "Bootstrap Aggregating" or "Bagging". The training set and their response is given by (1) and (2).

$$
\begin{array}{ll}
\text { Given training set } & \mathrm{T}=t_{1}, t_{2} \ldots t_{n} \\
\text { Response } & \mathrm{R}=r_{1}, r_{2} \ldots r_{n}
\end{array}
$$

Bagging repeatedly selects (B times) a random samples with replacement of the given training set.

$$
\text { For } b=1,2 \ldots B
$$

1. Sample with replacement, $\mathrm{n}$ training examples from $\mathrm{T}, \mathrm{R}$, call these as $T_{b}, R_{b}$.

2. Train a classification $f_{b}$ on $T_{b}, R_{b}$.

After training, predictions for unseen samples $t^{\prime}$ can be made by averaging the prediction from the entire individual regression tree ont' by equation (3). 


$$
\hat{\hat{f}}=\sum_{b=1}^{B} f_{b\left(t^{\prime}\right)}
$$

The succeeding step is "Feature Bagging", for correlation handling. If one or few elements are very powerful predictor for response variable, these elements will be chosen in many of the B trees, inflicting them to emerge as correlated, An evaluation of how bagging and random subspace make contributions to Accuracy features beneath one of a kind condition.

\section{IV.RESULTS AND DISCUSSION}

\section{Experimental Setup:}

Net Beans is used for this implementation with the aid of MySql and JSP. MySql is open source relational database administration system, used to gaining access to and managing the DB. MySql database is accessed by using many programming languages with language precise APIs consists of libraries .JSP (Java Server Pages) is a programming technological know-how in server facet used for creates a platformunbiased internet primarily based purposes and additionally helps builders to creates dynamically generated net pages primarily based on HTML, XML ,etc. An preliminary scan is performed to confirm the effectiveness of the proposed work in phrases of accuracy. Proposed algorithm works by means of realizing the remarks given by means of the cease users, with the assist of selection trees. Accuracy of classification is done by way of no of effective feedback and negatives comments. The proposed algorithm achieves $87 \%$ accuracy in the crowd sourcing classification, which is greater than that of the current algorithms.

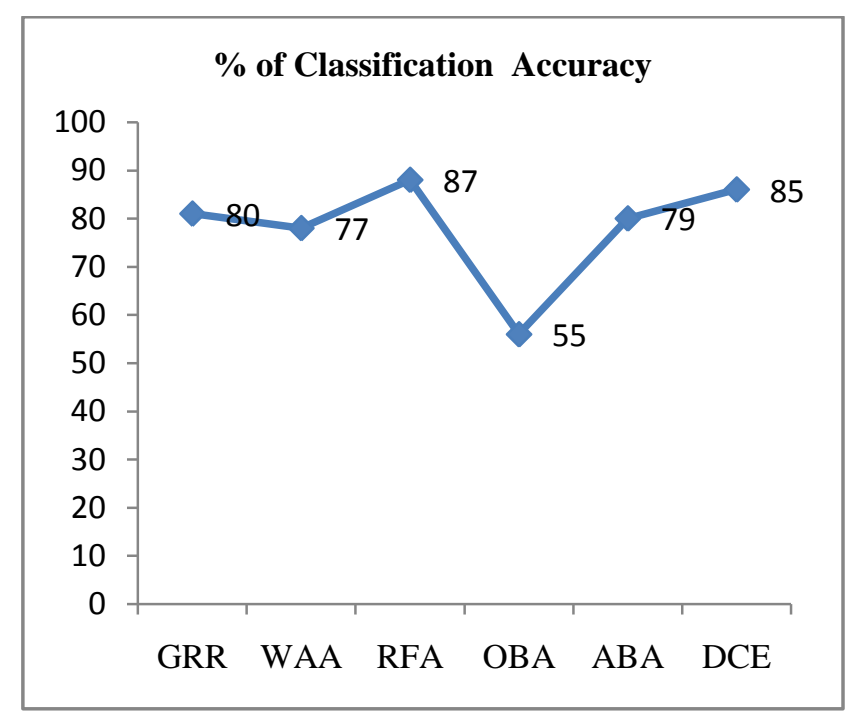

Figure 3: Comparison between RFA with other algorithms

The acronyms of the existing algorithms are justified in chapter II (Related Works). RFA is compared with these algorithms only on the basis of accuracy as shown in the Figure 3. 


\section{CONCLUSION}

Crowd Sourcing is acquiring the records or enter of a specific project given through the giant variety of people whom are worried in services, generally by using the Internet. The software of the classification tasks enables the increasing reputation of crowd-sourcing market. Random Forest Algorithm (RFA) used for classification in crowd-sourcing and enhancement of effectivity is executed through Dynamic Resource algorithm. This makes a first-class evaluation for classification in crowd-sourcing and improves the classification accuracy in crowd-sourcing applications. When in contrast to different algorithms, it achieves greater share of accuracy in crowd-sourcing classification. The categorised critiques are analyzed by using to confirm the first-rate of the product or items. The overall performance of the proposed work achieves a promising result of accuracy of 87 percentage. In future, it is proposed to gadget this framework for a range of functions in one of a kind crowd-sourcing platforms.

\section{VI.REFERENCES}

[1]. LibinZheng, Lei Chen,DLTA: A Framework for Dynamic Crowd sourcing Classification Tasks. VOL 31, NOV 5, MAY 2019.

[2].

ohammad Asghari, Cyrus Shahabi, On On-line Task Assignment in Spatial Crowd sourcing, 2017.

[3]. Xiao Bo, Christian Poellabauer, Detecting Label Errors in Crowd-Sourced Smartphone Sensor Data. International Workshop on Social Sensing, 2018.

[4]. Ting Wu, Chen Jason Zhang, Lei Chen, Pan Hui and Siyuan Liu, Object Identification with Pay-As-You-Go Crowd sourcing, 2016 IEEE International Conference on Big Data (Big Data).

[5].

XiaoniDuan, Keishi Tajima, Improving Classification Accuracy in Crowd sourcing through Hierarchical Reorganization,978-5386-2715-0/17, IEEE International Conference on Big data,2017.

[6]. Antonella Frisiello, Quynh Nhu Nguyen, Claudio Rossi, Fabrizio Dominici,GamifiedCrowd sourcing for Disaster Risk Management,2017 .

[7]. RaffaellaGuida, Peter T.B. Brett and Salman S. Khan,remote sensing and crowd sourcing, IEEE 978-1-4799-1114-1/3.

[8].

ien To, Seon Ho Kim, Cyrus Shahabi,Effectively Crowd sourcing the Acquisition and Analysis of Visual Data for Disaster Response, IEEE International Conference on Big Data (BIG DATA), 2015.

[9]. Dr.ShyamalaRamachandran, V. Sasireka2, Descriptive Study and Analysis of Crowd Sourcing Techniques in Mobile Social Media Networks, IEEE Transactions on Mobile Computing, 2017.

[10]. C.Bielski, V.O’Brien, C.Whitmore, K.Ylinen,I.Juga, Coupling Early Warning Services, Crowdsourcing, and Modelling for Improved Decision Support and Wildfire Emergency Management,2017.

[11]. NingXu, Jiangping Wang, An Ontological Bagging Approach for Image Classification of Crowd sourced Data, National Science Foundation Grant DBI 10-62351, and U.S.

[12]. Markus herb, Tobias weiherer, Nassir Navab, Federico Tombari, Crowd Sourced Semantic Edge mapping for autonomous vehicles, IEEE/RSJ International Conference on Intelligent Robots and Systems (IROS), 2019. 
[13]. Sumit Mishra, AkashYadav, Ashok Singh Sairam, Worker Selection in Crowd Sourced platforms using Non-dominated Sorting, 978-1-7281-1895-6/19,IEEE Region 10 Conference (TENCON), 2019.

[14].Xiangpeng wan, Hakim Ghazzai, YehiaMassoud, Real Time Navigation in Urban Areas Using Mobile Crowd Sourced Data, 978-1-5386-8396-5/19, IEEE International Systems Conference (SysCon), 2019 